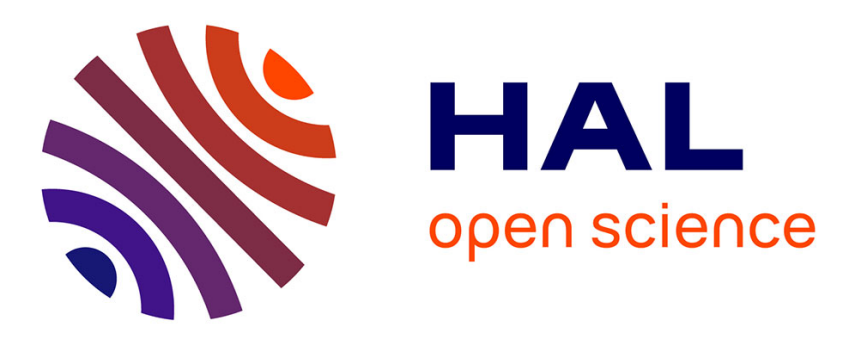

\title{
Estimating Vehicle Position Using Few Range Measurements to a Known Curved Surface
}

\author{
Michael Kokko, Franz Hover
}

\section{To cite this version:}

Michael Kokko, Franz Hover. Estimating Vehicle Position Using Few Range Measurements to a Known Curved Surface. 6th International Conference on Field and Service Robotics - FSR 2007, Jul 2007, Chamonix, France. inria-00202693

\section{HAL Id: inria-00202693 https://hal.inria.fr/inria-00202693}

Submitted on 7 Jan 2008

HAL is a multi-disciplinary open access archive for the deposit and dissemination of scientific research documents, whether they are published or not. The documents may come from teaching and research institutions in France or abroad, or from public or private research centers.
L'archive ouverte pluridisciplinaire HAL, est destinée au dépôt et à la diffusion de documents scientifiques de niveau recherche, publiés ou non, émanant des établissements d'enseignement et de recherche français ou étrangers, des laboratoires publics ou privés. 


\title{
Estimating Vehicle Position Using Few Range Measurements to a Known Curved Surface
}

\author{
Michael A. Kokko ${ }^{1}$ and Franz S. Hover ${ }^{2}$ \\ 1 Massachusetts Institute of Technology, Cambridge, MA kokkom@alum.mit.edu \\ 2 Massachusetts Institute of Technology, Cambridge, MA hover@mit.edu
}

Summary. This paper presents a method by which data from two or more rangefinders can be combined to estimate vehicle position when operating in proximity to a surface of known curvature. Estimates are combined in an Extended Kalman Filter which accounts for the expected transformation of sensor noise. Although developed for use with Autonomous Underwater Vehicles, this curvature-based localization technique can be applied to any mobile agent capable of measuring its range to a previously-mapped surface.

\section{Introduction}

Many autonomous vehicles rely on navigation data obtained from absolute position and orientation sensors including GPS units and compasses; however, it is often necessary to operate vehicles in environments that preclude the use of such sensors. Our present application, for example, involves inspecting ship hulls with an Autonomous Underwater Vehicle (AUV) in harbor environments where neither GPS nor compass readings are available. Various sensor-based navigation strategies are possible for the ship hull inspection task. One approach is to deploy and calibrate a network of transponders to provide localization information [10]. Another approach is to use acoustic or optical images to estimate the 3D structure of the ship hull and concurrently estimate vehicle pose $[1,2,8]$. Our prototype inspection vehicle currently obtains odometry information by means of a four-beam Teledyne RDI Doppler Velocity Log (DVL) sensor which tracks relative motion between the AUV and its target hull.

In this paper ${ }^{3}$, we propose an alternative method of navigation based on a small number of range measurements taken to a known surface. We envision this capability to provide on-line, real-time servoing in close proximity to

\footnotetext{
${ }^{3}$ This work and the development of the Hovering Autonomous Underwater Vehicle have been supported by the Office of Naval Research under grant N00014-06-10043 which is monitored by Dr. T.F. Swean.
} 
curved sections of target ship hulls. Similar work has been done by Madhavan and Durrant-Whyte [6] in which they navigate based on points of maximum curvature extracted from observed surfaces. Several other authors including Sekmen [7] have considered the problem of identifying the location and curvature of cylindrical targets from a mobile agent. Kondo and Ura also use curvature in [5] to track features with their AUV remaining at a desired relative heading.

The present approach differs from these in that we produce actual vehicle position estimates by exploiting the known geometric properties of an observed surface. Details of the observation geometry are considered and the derived position estimates are integrated into an Extended Kalman Filter (EKF) framework. Both simulated navigation results as well as data obtained from a real-time surface craft implementation are presented.

\section{Vehicle Platform}

The Hovering Autonomous Underwater Vehicle (HAUV) in this work measures roughly $1.0 \mathrm{~m} \times 0.7 \mathrm{~m} \times 0.4 \mathrm{~m}$ and weighs approximately $80 \mathrm{~kg}$. More detailed descriptions of the physical system are presented in [9] (see also Fig. 1). Equipped with both a DVL and a Sound Metrics DIDSON imaging sonar, the HAUV has been designed to servo laterally along a ship hull, inspecting its surface for the presence of mines. Since both compass and GPS signals are useless in these conditions, the HAUV integrates velocity estimates derived from DVL beam measurements to track its position along the hull. Meanwhile, the DIDSON captures high-resolution imagery which can later be reconstructed in the form of a mosaic to view the entire inspection area.
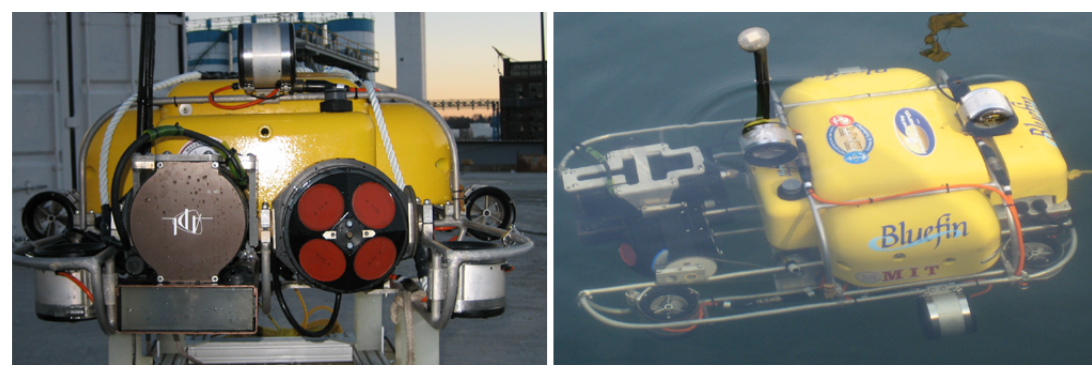

Fig. 1. The HAUV with its sensor payload comprised of a DIDSON imaging sonar (left sensor) and a DVL (right sensor). 


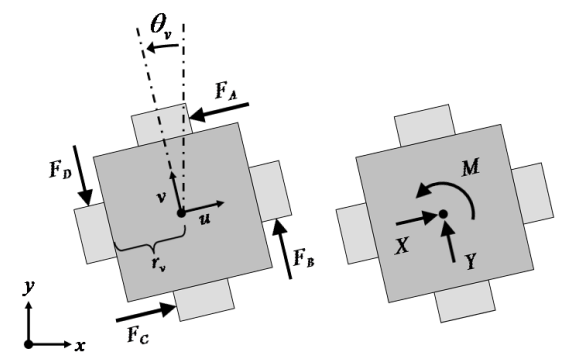

Fig. 2. Free-body diagram of the simplified HAUV dynamic model indicating chosen thrust and yaw conventions.

\section{Dynamic Model}

A state-space model of a simplified HAUV-like system can be developed for use in simulation. The vehicle states are taken to include vehicle-relative velocities (two planar and one rotational) as well as global Cartesian locations and vehicle yaw. That is:

$$
\mathbf{x}(t)=\left[\begin{array}{llllll}
u & v & \dot{\theta_{v}} & x_{v} & y_{v} & \theta_{v}
\end{array}\right]^{T}
$$

At low velocities, when the effects of hydrodynamic drag on the system are negligible, the system dynamics propagate as:

$$
\begin{aligned}
\dot{\mathbf{x}}(t) & =\mathbf{f}\left(\mathbf{x}(t), \mathbf{u}_{\mathbf{v}}(t), t\right)+\mathbf{w}(t) \\
\mathbf{z}_{\mathbf{k}} & =\mathbf{h}\left(\mathbf{x}\left(t_{k}\right)\right)+\mathbf{v}_{\mathbf{k}}
\end{aligned}
$$

In this representation, $\mathbf{x}(t)$ are the vehicle states, $\mathbf{u}_{\mathbf{v}}(t)$ are control inputs $\{X, Y, M\}, \mathbf{z}_{\mathbf{k}}$ are measurements, and $\mathbf{w}(t)$ and $\mathbf{v}_{\mathbf{k}}$ add Gaussian noise with respective variances of $\mathbf{Q}$ and $\mathbf{R}_{\mathbf{k}}$. Notice that the vehicle state vector propagates continuously while measurements are made in discrete time. The nonlinear state propagation function $\mathbf{f}\left(\mathbf{x}(t), \mathbf{u}_{\mathbf{v}}(t), t\right)$ can be defined with respect to vehicle mass $(m)$ and inertia $(J)$ as:

$$
\begin{gathered}
\mathbf{f}\left(\mathbf{x}(t), \mathbf{u}_{\mathbf{v}}(t), t\right)=\left[X / m Y / m M / J c_{1} c_{2} \dot{\theta}_{v}\right]^{T} \\
\text { where: } \\
\qquad \begin{array}{r}
c_{1}=u \cos \theta_{v}-v \sin \theta_{v} \\
c_{2}=u \sin \theta_{v}+v \cos \theta_{v}
\end{array}
\end{gathered}
$$

A simple PD control scheme drives the estimated state $\hat{\mathbf{x}}(t)$ to a reference state $\mathbf{x}_{\mathbf{r}}(t)$ by means of an error vector $\tilde{\mathbf{x}}(t)=\mathbf{x}_{\mathbf{r}}(t)-\hat{\mathbf{x}}(t)$. The controller was developed with dynamics linearized about the hovering condition which provides a means for selecting an adequate compensator of the form $\mathbf{u}_{\mathbf{v}}(t)=$ $-\mathbf{K}_{\mathbf{c}} \tilde{\mathbf{x}}(t)$. Proportional and derivative control gains were chosen to place the compensated system poles such that the $\theta_{v}$ step response settles in roughly 
0.5 seconds while the $x_{v}$ and $y_{v}$ responses settle about four times slower (each with minimal overshoot). These selections are consistent with the dynamic performance of the actual vehicle in operation.

\section{Observation Model}

Our approach to curvature-based navigation requires two or more rangemeasurements taken at fixed agent-relative angles. On the HAUV, these range measurements can be made by pitching the DVL down $22^{\circ}$ to emulate a pair of range-finding beams which lie in-plane with the vehicle and are separated by $41.4^{\circ}$. We assume that an accurate yaw position $\theta_{v}$ is available, at least in the short term, from an inertial measurement unit ${ }^{4}$, and that range measurements are taken between the agent and a target of known parabolic $\left(y=A_{w} x^{2}+B_{w}\right)$ curvature. With these assumptions, two range measurements $\left\{r_{a}, r_{b}\right\}$ taken at bearings $\left\{\theta_{a}, \theta_{b}\right\}$ are sufficient to produce an estimate of vehicle planar position $\left\{x_{v}, y_{v}\right\}$. Note that although $A_{w}$ is negative in Fig. 3 to model a parabolic pocket, there is no algorithmic difference when using a positive value corresponding to a protrusion as might be found on a ship hull.

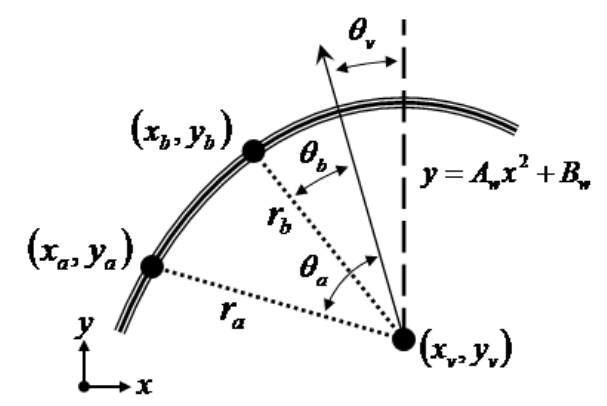

Fig. 3. Observation geometry modeled to simulate range data received from the DVL sensor on the HAUV. For the DVL case, $\theta_{a}=+20.7^{\circ}$ and $\theta_{b}=-20.7^{\circ}$.

$$
\begin{gathered}
x_{v}=\frac{r_{a} c_{a} / A_{w}-r_{b} c_{b} / A_{w}-r_{a}^{2} s_{a}^{2}+r_{b}^{2} s_{b}^{2}}{-2 r_{a} s_{a}+2 r_{b} s_{b}} \\
y_{v}=\frac{1}{2}\left\{A_{w}\left[\left(x_{v}-r_{a} s_{a}\right)^{2}+\left(x_{v}-r_{b} s_{b}\right)^{2}\right]+2 B_{w}-r_{a} c_{a}-r_{b} c_{b}\right\}
\end{gathered}
$$

where:

$$
\begin{aligned}
& s_{a}=\sin \left(\theta_{v}+\theta_{a}\right) s_{b}=\sin \left(\theta_{v}+\theta_{b}\right) \\
& c_{a}=\cos \left(\theta_{v}+\theta_{a}\right) c_{b}=\cos \left(\theta_{v}+\theta_{b}\right)
\end{aligned}
$$

\footnotetext{
${ }^{4}$ If only rate gyros are available, vehicle yaw position must be calibrated with respect to a known feature prior to generating position estimates.
} 
While only two range measurements are needed to develop a position estimate, it is clear that this procedure can be applied to the general $n$-beam scenario where $n \geq 2$. When redundant range information $(n>2)$ is available, $m=$ $\frac{n !}{2(n-2) !}$ distinct estimates of vehicle position can be combined by filtering. In this case, the observation vector at timestep $k$ becomes:

$$
\mathbf{z}_{\mathbf{k}}=\left[\begin{array}{lllll}
x_{v 1} & y_{v 1} & \cdots & x_{v m} & y_{v m} \\
\theta_{v}
\end{array}\right]^{T}
$$

The multi-beam sensor scenario is, in fact, a simplified version of scanning sonar observation. The algorithm presented here is directly applicable to the scanning case, allowing vehicle position estimates to be developed when these more advanced sensors are employed in environments of known curvature. It is also worth noting that this localization technique may still be applicable when features cannot be adequately described by a single parabolic curve. Estimation equations similar to (5) and (6) have been developed for sinusoidal pockets for example, and the same procedure can be applied to other types of curvature. Additionally, multiple curves can be combined in a piecewise manner to facilitate vehicle navigation along complex targets like ship hulls. In some cases the correct estimate my need to be selected from a set of candidate positions based on recent navigation history or an explicit switching metric.
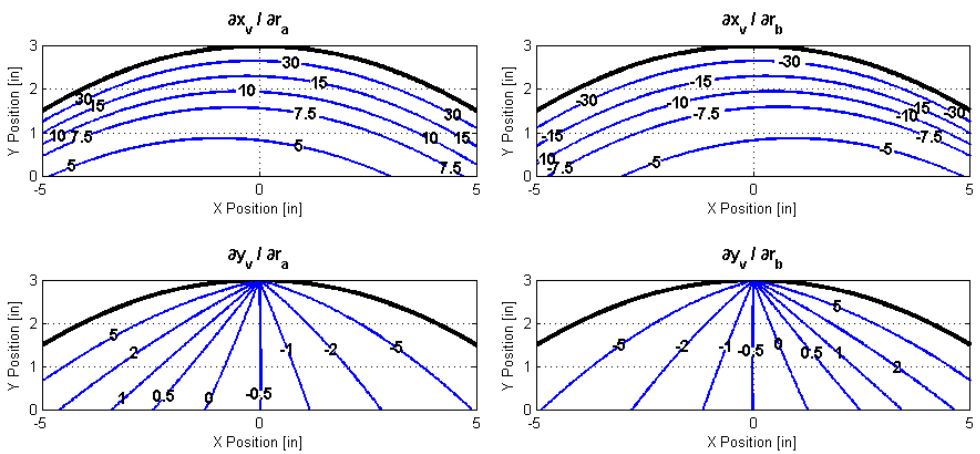

Fig. 4. Partial derivatives of estimated vehicle position shown for an agent with no yaw and a DVL-like sensor configuration (two symmetric range-finder beams separated by $41.4^{\circ}$ ) observing a surface conforming to the equation $y=-0.06 x^{2}+3$.

In addition to the geometric constraints derived in (5) and (6), it is also important to understand the noise properties of the resulting position estimate. By calculating the partial derivatives of these equations with respect to each range measurement we observe how sensor noise translates into estimate uncertainty. Figs. 4 and 5 show graphical representations of these derivatives which indicate that the noise on $x_{v}$ depends strongly upon the distance from the wall while the noise gradient on $y_{v}$ is much more gentle throughout. Notice 

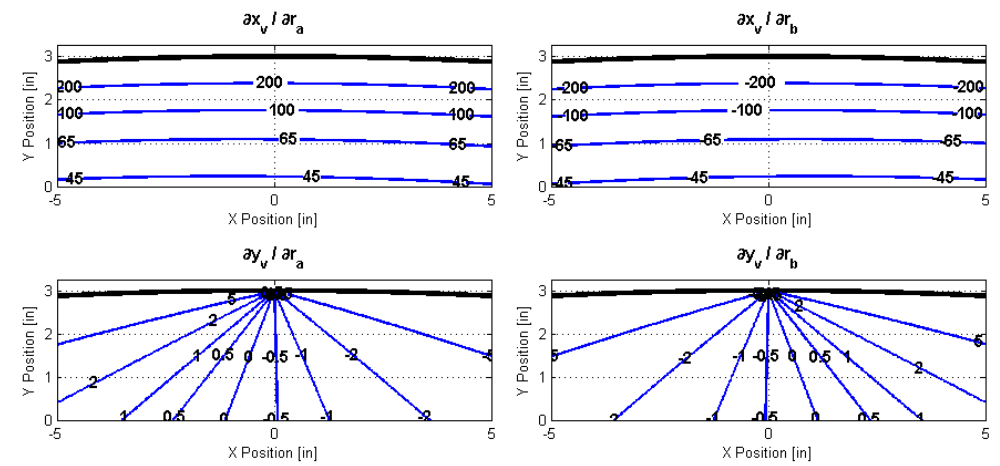

Fig. 5. Partial derivatives as in Fig. 4 with a target fitting $y=-0.005 x^{2}+3$. Notice that the horizontal position estimate noise greatly increases as the target surface flattens.

that the noise on $x_{v}$ becomes substantially higher when the surface flattens. In Fig. 5, for example, the vehicle would have to move roughly $26 \mathrm{~m}$ away from the wall to achieve $\partial x_{v} / \partial r_{a}$ and $\partial x_{v} / \partial r_{b}$ values of \pm 5 which occur at a range of only $2 \mathrm{~m}$ in Fig. 4. Thus, this navigation scheme is most useful in situations where highly curved structures can be observed at an appropriate distance.

\section{Nonlinear Estimator}

An Extended Kalman Filter can be employed to combine estimates developed based on the observations in $\mathbf{z}_{\mathbf{k}}$. At each timestep, the filtering process begins by calculating the Jacobian of the nonlinear state propagation function $\mathbf{F}(\mathbf{x}(t), t)$ as well as the Jacobian of the observation model $\mathbf{H}_{\mathbf{k}}$ using (8) and (9), respectively. Notice that the first two rows of the observation Jacobian are repeated $m$ times, where $m$ reflects the number of range-pair-based estimates included in $\mathbf{z}_{\mathbf{k}}$ (which itself has $2 m+1$ rows as seen in (7)).

$$
\mathbf{F}(\mathbf{x}(t), t)=\left[\begin{array}{cccccc}
0 & 0 & 0 & 0 & 0 & 0 \\
0 & 0 & 0 & 0 & 0 & 0 \\
0 & 0 & 0 & 0 & 0 & 0 \\
\cos \theta_{v} & -\sin \theta_{v} & 0 & 0 & 0 & c_{3} \\
\sin \theta_{v} & \cos \theta_{v} & 0 & 0 & 0 & c_{4} \\
0 & 0 & 1 & 0 & 0 & 0
\end{array}\right]
$$

where:

$$
\begin{gathered}
c_{3}=-u \dot{\theta}_{v} \sin \theta_{v}-v \dot{\theta}_{v} \cos \theta_{v} \\
c_{4}=u \dot{\theta}_{v} \cos \theta_{v}-v \dot{\theta}_{v} \sin \theta_{v}
\end{gathered}
$$




$$
\mathbf{H}_{\mathbf{k}}=\left[\begin{array}{cccccc}
0 & 0 & 0 & 1 & 0 & 0 \\
0 & 0 & 0 & 0 & 1 & 0 \\
0 & 0 & 0 & 1 & 0 & 0 \\
0 & 0 & 0 & 0 & 1 & 0 \\
\vdots & \vdots & \vdots & \vdots & \vdots & \vdots \\
0 & 0 & 0 & 0 & 0 & 1
\end{array}\right]
$$

With the Jacobians calculated, a priori state $\hat{\mathbf{x}}_{\mathbf{k}}{ }^{-}$and error covariance $\mathbf{P}_{\mathbf{k}}{ }^{-}$ estimates can be generated by means of the standard continuous-discrete EKF formulation found in [3]. When new curvature-based position estimates are available in $\mathbf{z}_{\mathbf{k}}$, a Kalman gain can be calculated and the a priori estimates updated. The predicted observation vector $\mathbf{h}_{\mathbf{k}}\left(\hat{\mathbf{x}}_{\mathbf{k}}{ }^{-}\right)$and the observation noise covariance $\mathbf{R}_{\mathbf{k}}$ are dependent upon vehicle position and must be recomputed at each timestep.

$$
\begin{aligned}
\mathbf{h}_{\mathbf{k}}\left(\hat{\mathbf{x}}_{\mathbf{k}}{ }^{-}\right)= & {\left[\hat{x}_{v} \hat{y}_{v} \cdots \hat{x}_{v} \hat{y}_{v} \hat{\theta}_{v}\right]^{T} } \\
\mathbf{R}_{\mathbf{k}} & =\left[\begin{array}{cccc}
R_{1,1} & \cdots & R_{1,2 m} & 0 \\
\vdots & \ddots & \vdots & \vdots \\
R_{2 m, 1} & \cdots & R_{2 m, 2 m} & 0 \\
0 & \cdots & 0 & \sigma_{\theta_{v}}^{2}
\end{array}\right]
\end{aligned}
$$

where:

$$
R_{i, j}=\frac{\partial z_{i}}{\partial r_{a}} \frac{\partial z_{j}}{\partial r_{a}} \sigma_{r_{a}}^{2}+\frac{\partial z_{i}}{\partial r_{b}} \frac{\partial z_{j}}{\partial r_{b}} \sigma_{r_{b}}^{2}
$$

In summary, the curvature-based estimation algorithm follows a simple procedure at each timestep $(\mathrm{k})$ where new range data is available:

1. Calculate Jacobians with (8) and (9) and perform the EKF prediction step propagating the state and covariance estimates forward.

2. Capture a set of $n$ range measurements taken between the mobile agent and a surface of known curvature.

3. Calculate a set of $m$ agent position estimates $\left\{x_{v i}, y_{v i}\right\}$ using (5) and (6) where each estimate is based on a unique pair of range measurements. These positions are a subset of $\mathbf{z}_{k}$ as defined in (7).

4. Construct a predicted observation vector $\mathbf{h}_{\mathbf{k}}\left(\hat{\mathbf{x}}_{\mathbf{k}}{ }^{-}\right)$as in (10) using the predicted state and compute the observation covariance matrix $\mathbf{R}_{\mathbf{k}}$ with (11).

5. Calculate the Kalman gain and perform the EKF correction step to generate new state and error covariance estimates.

\section{Simulation Results}

The performance of the simplified dynamic system developed in Section 3 with curvature-based observations provided to an EKF has been simulated for a 
variety of target and beam configurations. Sensor readings were taken in these simulations discretely at $10 \mathrm{~Hz}$ as is typical for a DVL, and both the actual vehicle state and the EKF were propagated continuously via the Runge-Kutta method between timesteps. Range sensor noise standard deviations of $6 \mathrm{~mm}$ were assumed, as was a nominal yaw sensor deviation of 0.005rad. Diagonal elements of the $\mathbf{Q}$ matrix representing $X, Y$, and $M$ variances of $(5 \mathrm{~N})^{2},(4 \mathrm{~N})^{2}$ and $(0.5 \mathrm{Nm})^{2}$ were chosen to ensure a faster response from the estimator than from the controller.

Fig. 6 presents simulation results which indicate that relatively accurate trajectory-following is feasible using curvature-based navigation. As expected, position errors in the $x_{v}$ direction are larger in magnitude than those in the $y_{v}$ direction $\left(\sigma_{x, e r r}=2.9 \mathrm{~cm}\right.$ vs $\left.\sigma_{y, e r r}=1.1 \mathrm{~cm}\right)$ due to the increased sensitivity predicted by Figs. 4 and 5 . Since position estimate noise rapidly increases close to the observed surface, better results can be obtained by operating farther from the wall.

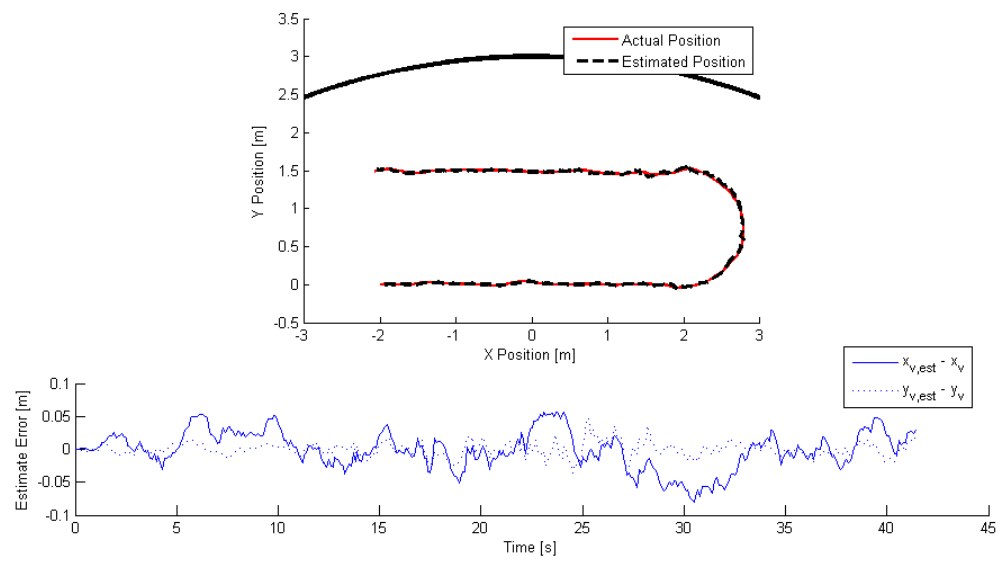

Fig. 6. Results of a typical simulation with no vehicle yaw, two sensor beams spaced $41.4^{\circ}$ apart, and range error deviations of $1 \mathrm{~mm}$. The vehicle followed a ' $U^{\prime}$-shaped path from the lower-left at approximately $0.25 \mathrm{~m} / \mathrm{s}$.

\section{Real-Time Implementation}

A small raft robot equipped with a Hokuyo URG miniature laser scanner was constructed to validate the range-based navigation procedure prior to implementation on the HAUV. Two range measurements separated by $41.4^{\circ}$ were taken to a parabolic wall fitting the equation ${ }^{5} y=-0.015 x^{2}$ and all data

\footnotetext{
${ }^{5}$ The units of $A_{w}$ are $\left[\mathrm{in}^{-1}\right]$ for the target employed in this experiment.
} 
from the laser were used to determine vehicle yaw. A fully discrete EKF was implemented in $\mathrm{C}++$ on a Mac Mini PC and integrated into a control loop running at $2.5 \mathrm{~Hz}$ (limited by sensor communication latencies). This relatively slow sampling rate bounded the bandwidth of both the PD controller and the estimator, leading to long response and settling times.

The raft ran a number of missions during which it transited to and held station at $(0 \mathrm{~m},-0.635 \mathrm{~m})$ relative to the target vertex. Results from a typical test are shown in Fig. 7. During the final 50 seconds of this mission the $x$ and $y$ estimate standard deviations are observed to be $8.1 \mathrm{~mm}$ and $4.1 \mathrm{~mm}$ (respectively) which are reasonably close to the predicted deviations of $8.9 \mathrm{~mm}$ and $2.4 \mathrm{~mm}$ calculated using (11). This result confirms that localization accuracy is indeed a function target-relative agent pose as shown in Figs. 4 and 5. The magnitude of the experimental estimate noise is significantly smaller than the simulated results shown in Fig. 6 due in part to more accurate laser scanner range measurements $\left(\sigma_{r}=3.3 \mathrm{~mm}\right)$.

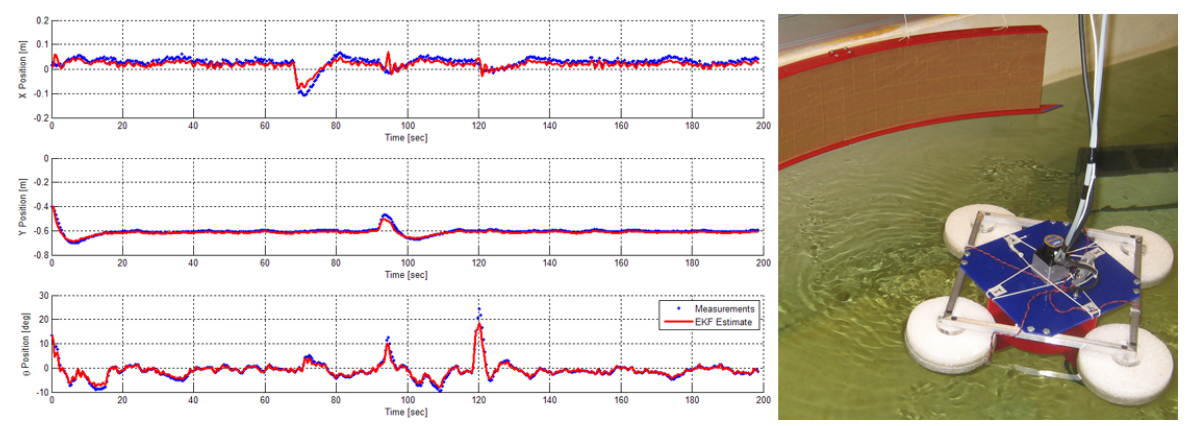

Fig. 7. The raft robot servoing relative to a parabolic target. Data from this 200second test demonstrate the system's ability to hold station and recover from $x, y$, and $\theta$ disturbances.

\section{Conclusions and Future Work}

Extending curvature-based localization to underwater applications using sonar range measurements will necessarily involve an examination of acoustic reflection from curved structures. It is expected that high-frequency, narrow-beam sonar systems similar to the DVL will provide the most accurate measurements. The navigation scheme itself is expected to prove useful in situations where sections of the target hull can be modeled with reasonable accuracy prior to inspection (e.g. from ship's lines); however, its effectiveness will diminish as the hull curvature flattens as viewed from the sensing position unless 
we move away a commeasurate amount. Potential extensions of this work including multi-curve and three-dimensional navigation are discussed in [4].

Although more advanced image and scanning sonar based navigation techniques have been widely adopted, the present curvature-based solution is attractive because of its simplicity, minimal computational requirements, and application to navigation in truly hostile environments. The algorithm presented in this paper relies on a minimum of two range measurements which need not always be provided by a DVL as we consider here; rather, this method can be implemented on nearly any planar land, air, or undersea craft equipped with at least two range-finders and a yaw sensor. The curvature-based localization technique can either serve as a redundant pose estimator to more complex scanning techniques or it can assume full localization responsibility in situations where more sophisticated sensors are inoperable, e.g., replacing a vision-based system in dark or murky environments.

\section{References}

1. R. Eustice, O. Pizarro, and H. Singh, "Visually Augmented Navigation in an Unstructured Environment Using a Delayed State History," in Proceedings of the 2004 IEEE International Conference on Robotics and Automation, vol. 1, New Orleans, USA, April 2004, pp. 25-32.

2. H. J. S. Feder, J. J. Leonard, and C. M. Smith, "Adaptive mobile robot navigation and mapping," The International Journal of Robotics Research, vol. 18, no. 7, July 1999.

3. A. Gelb, Ed., Applied Optimal Estimation. Cambridge: The M.I.T. Press, 1974.

4. M. Kokko, "Range-based navigation of AUVs operating near ship hulls," Master's thesis, Massachusetts Institute of Technology, 2007.

5. H. Kondo and T. Ura, "Navigation of an AUV for investigation of underwater structures," Control Engineering Practice, vol. 12, no. 12, pp. 1551-1559, December 2004.

6. R. Madhavan and H. F. Durrant-Whyte, "Natural landmark-based autonomous vehicle navigation," Robotics and Autonomous Systems, vol. 46, no. 2, pp. 79-95, February 2004.

7. A. S. Sekmen and B. Barshan, "Estimation of object location and radius of curvature using ultrasonic sonar," Applied Acoustics, vol. 62, no. 7, pp. 841865, July 2001.

8. J. D. Tardos, J. Neira, P. M. Newman, and J. J. Leonard, "Robust Mapping and Localization in Indoor Environments Using Sonar Data," The International Journal of Robotics Research, vol. 21, no. 4, pp. 311-330, 2002. [Online]. Available: http://ijr.sagepub.com/cgi/content/abstract/21/4/311

9. J. Vaganay, M. Elkins, D. Esposito, W. O'Halloran, F. Hover, and M. Kokko, "Ship Hull Inspection with the HAUV: US Navy and NATO Demonstration Results," in Proceedings of IEEE / MTS Oceans 2006, 2006.

10. L. Whitcomb, D. Yoerger, and H. Singh, "Towards Precision Robotic Manuvering, Survey and Manipulation in Unstructured Undersea Environments," in Robotics Research - The Eighth International Symposium, Springer Verlag, London, 1998, pp. 45-54. 